Comunicación popular, historia y medios comunitarios

Felipe Navarro Nicoletti

Question, Vol. 1, N. 56, e012, octubre-diciembre 2017. ISSN 1669-6581

http://perio.unlp.edu.ar/ojs/index.php/question/article/view/4369

IICom- FPyCS | Universidad Nacional de La Plata

La Plata | Buenos Aires | Argentina

Recibido: 21-09-2017 Aceptado: 16-11-2017

Cita sugerida: Navarro Nicoletti, F. (2017). Comunicación popular, historia y medios comunitarios. Question, 1(56),

e012. doi: https://doi.org/10.24215/16696581e012

\title{
Comunicación popular, historia y medios comunitarios
}

\author{
Popular communication, history and community media
}

Felipe Navarro Nicoletti

Instituto de Investigaciones en Diversidad Cultural y Procesos

de Cambio; Universidad Nacional de Río Negro/ Agencia

Nacional de Promoción Científica y Tecnológica (Argentina)

fnavarro.nicoletti@gmail.com

\section{Resumen}

En el artículo ponemos en discusión categorías que al día de hoy pueden parecer puras y cerradas. La comunicación en general y la comunicación comunitaria en su particularidad, el rol del periodista y el del comunicador comunitario en clave de vínculo prolongado en el tiempo con la comunidad en la que se encuentra inserta, son conceptos que definimos y cruzamos, buscando diferencias y similitudes. Unimos lo anteriormente definido en el caso de la radio comunitaria Encuentro, ubicada en la ciudad de Viedma, Río Negro. Este medio da cuenta de un trabajo periodístico con enfoque comunitario y nos ayuda a trazar un paralelismo con lo conceptualmente definido. 
Palabras clave: comunicación; comunicación comunitaria; periodismo; radiodifusión local.

\begin{abstract}
In the article we put in discussion categories that today may seem pure and closed. The Communication in general and community communication in its particularity, the role of the journalist and community communicator in terms of link prolonged in time with the community in which is inserted, are concepts that we define and crossed, looking for differences and similarities. We unite defined above in the case of community radio Encuentro, located in the city of Viedma, Río Negro. This media realizes a journalistic work with community approach and helps us draw a parallel with conceptually defined.
\end{abstract}

Keywords: communication; community communication; journalism; local broadcasting.

En el siguiente trabajo tenemos como objetivo principal dar cuenta de categorías generales como las de comunicación y comunicación comunitaria, pasando por la del periodista y comunicador comunitario apoyándonos en un caso ejemplificador que nos permita dar cuenta de la necesidad de un trabajo complementario en el marco de la inserción social. Si bien pretendemos manifestar especificaciones de cada concepto, nuestro objetivo radica en poder demostrar la necesidad de una relación complementaria para lograr el impacto, la participación y la transformación social. Para el armado, efectuamos un análisis bibliográfico pertinente para los conceptos a desarrollar y una entrevista en profundidad a la referente de la radio Encuentro (caso ejemplificador), María Cristina Cabral, que nos proporcionó información para desarrollar el caso y anclarlo con la teoría seleccionada.

Teniendo en consideración que la comunicación es ineludible para la satisfacción de necesidades humanas y desarrollo social, podemos dar cuenta que la misma atraviesa todos los ámbitos de la vida social. De esta manera se conforman innumerables modos y medios para canalizar los flujos de comunicación. Como un modo más de entender a la sociedad en la que habitamos, la comunicación comunitaria surge desde diversos procesos culturales y sociales, donde la participación colectiva abre la posibilidad de nodos comunicacionales que responden específicamente a los intereses de los sectores populares.

Los procesos comunicacionales son socialmente conocidos, principalmente, por el desarrollo constante de medios de comunicación que facilitan la corriente dinámica de información. Históricamente en la Argentina, los procesos comunicacionales intervenidos por los medios de comunicación se establecieron desde soportes que respondían y responden a un fin lucrativo, 
privado, masivo y hegemónico. Ante numerosos episodios de atropellos y exclusión social, los medios de comunicación comunitarios surgieron para exponer estos hechos y para poder "darle voz" a sectores que estaban excluidos y no eran atendidas sus demandas (Mac Bride, 1980). La comunicación popular y alternativa genera un espacio histórico donde los sectores excluidos tienen la posibilidad de apropiarse de su propio entorno y comunicarlo como lo crean conveniente, generando un espacio de construcción comunitaria desde su propio entorno.

La primera ley (1) que reguló los medios de comunicación en Argentina poseía dos horizontes bien demarcados: el nuevo orden económico basado en la acumulación de capitales, reestructurando toda la dinámica social del país y la persecución contra lo que las fuerzas represivas consideraban subversivo (Postolsky y Marino, 2005); relegando la participación a cualquier expresión mediática que no encajara en el proceso productivo nacional .Las radios comunitarias, canal en el que centraremos el siguiente trabajo, nacen en un contexto de exposición de los atropellos a la libre comunicación y de visibilización de un sector históricamente relegado por los medios de comunicación hegemónicos. Los medios de comunicación comunitarios comenzaron a crecer exponencialmente hasta llegar a ser contemplados por la Ley de servicios de comunicación audiovisual 26522, sancionada en 2009 (2).

Bajo el contexto en el que los medios de comunicación comunitarios buscan un lugar en el espectro mediático, ya cooptado por los grupos hegemónicos de comunicación, surge la figura del comunicador comunitario. A esta figura que en el siguiente trabajo intentaremos describir, la ponemos en debate con la figura del periodista, pudiendo encontrar similitudes y diferencias frente a la construcción de la noticia, su difusión y relación con su posible público, en tanto ejercicio profesional. Uno de los aspectos que nos parece clave destacar, al momento de introducir este debate, es la relación o no con la comunidad a la que se dirigen como comunicadores. Queda claro que para que haya una comunicación, teóricamente hablando, tiene que existir relación entre las condiciones de producción, discurso y las condiciones de reconocimiento para que el mensaje pueda ser decodificado de una cierta manera. La clave diferencial del comunicador comunitario con el periodista subyace en el poder de interrelación directa con su receptor.

El trabajo contará con tres apartados principales. El primero se desarrollará de modo introductorio en relación a la comunicación en general, derivando en la comunicación comunitaria en tanto surgimiento, razón de ser y sus principales campos de intervención. En un segundo apartado focalizaremos el análisis en la figura del comunicador comunitario, pudiendo establecerse una suerte de comparación con la figura del periodista bajo categorías específicas de las dinámicas comunicacionales. En ambos apartados haremos hincapié en el soporte radiofónico, para poder establecer una ejemplificación de todos los procesos mencionados y a la vez no extendernos en el análisis de todos los canales de comunicación. Por último, 
expondremos el caso de radio Encuentro, ubicada en la ciudad de Viedma, que nos permite evidenciar lo expuesto en los primeros dos apartados.

\section{La comunicación cotidiana y participativa}

En todos los aspectos de nuestra vida cotidiana hay comunicación, en todo acto que realizamos establecemos una acción comunicativa, para la cual, como afirma Habermas (1981), sólo se necesitan dos sujetos capaces del lenguaje. No podemos escapar de ella, pero sí manipularla (3) de manera que nos pueda ser útil para ciertos objetivos. "La comunicación, por tanto, constituye un medio indiscutible para poder crecer personal y socialmente, facilita a una sociedad o grupo social el crecimiento y desarrollo en el plano cultural y económico" (Sánchez, 2002: 107). Podemos afirmar que la evolución de las sociedades va de la mano de procesos comunicacionales que exponen diversas realidades a lo largo del tiempo. Los medios de comunicación fueron facilitando a lo largo de la historia la globalización de la información y la cultura, así como la formación de ciertos patrones culturales y sociales que derivan en la dinámica social que nos envuelve.

Es así como podemos dar cuenta que "la comunicación es parte integral y no se puede comprender por fuera de las prácticas sociales que protagonizan los sujetos en la historia" (Uranga y Thompson, 2016: 39). Esta pasa a ser un elemento fundamental y fundacional en el funcionamiento interno de una sociedad. La ampliación de los medios de comunicación social facilita las mediaciones sociales, como la promoción al debate de lo instituido. "Nadie puede exigir algo que no reconoce como suyo y ninguna demanda trasciende si no es a través de la producción de mensajes que luego pueden transformarse en políticas culturales" (Uranga, 2016: 62). Para expandir el campo de incidencia de la comunicación, la transformación social y la democratización de la palabra, hace falta una sociedad que se involucre en la producción de mensajes, que pueda apropiarse de su entorno para resignificarlo y no sólo ser espectadora pasiva.

Podemos asegurar que la comunicación nos atraviesa por completo, resta elegir si estamos dispuestos a dejarnos llevar por el flujo social que se nos impone, o poder pensar los procesos comunicacionales desde la transformación social. Aquí es cuando invitamos a repensar la comunicación desde una perspectiva popular y comunitaria, que nos permita un contacto más fiel y directo con las demandas sociales. "Los procesos comunicacionales tienen que contribuir a la movilización social entendida como procesos de empoderamiento político cultural" (Uranga, 2016: 68), poder entender la dinámica comunicacional de manera relacional, generando redes e intercambios, produciendo sentidos y construyendo la cultura en la que vivimos y pensamos. Ser receptores pasivos nos lleva a una dinámica de dominación y 
pensamiento abstracto en el sentido amplio de la palabra. Cuando hablamos de ampliación de la comunicación, más allá de invitar a mirar la misma desde lo más cotidiano, es también alejarnos de la "mirada reduccionista que limita la comunicación a los medios" (Cardoso, 2006: 3), que nos aleja de la mirada comunicacional desde la participación y la relación entre los actores sociales para su progreso social.

Sandra Massoni afirma que "hay que pensar el derecho a la comunicación como un derecho ampliado y relacionado con el conjunto de los derechos humanos" (Uranga, 2016: 11).Puesto que organismos internacionales como la UNESCO declaran el derecho a la comunicación como un derecho humano fundamental para el libre desarrollo social, pensar la comunicación simplemente desde los medios o la información que nos transmiten seria condenarnos a un futuro limitando nuestro propio derecho humano a comunicarnos libremente al encasillamiento mediático.

"Un enfoque de comunicación comunitaria, indica que los medios son medios, no fines en sí mismos" (Cardoso, 2006: 4); todo medio de comunicación comunitario surge desde la demanda, la necesidad y la problemática más local. Tenemos que poder generar escenarios en donde la comunicación sea un proceso habilitante para la construcción de actores en democracia (Balán, Jaimes, Alegría y Borri, 2013: 11). Es necesario forjar el espacio y el modo de canalizar lo más inmediato y cotidiano que nos sucede, sin tener que esperar a figurar en la agenda de grandes medios de comunicación que no abarca esos espacios. Martín-Barbero repiensa la comunicación en clave de mediaciones más que de medios, para así poder "re-ver el proceso entero de la comunicación desde su otro lado, el de la recepción, el de las resistencias que ahí tienen un lugar, el de la apropiación desde sus usos" (1987: 10).

La comunicación comunitaria nos permite establecer un diálogo donde las demandas y la voz de la comunidad local son el motor de la información para medios comunitarios y locales, en primera instancia. Estableciendo un proceso circular de la comunicación, donde la figura del emisor y receptor sean móviles y dinámicas. Para que se genere dicho proceso, es clave la participación. Cuando hablamos de participación, nos referimos a la "acción desarrollada por los miembros de la comunidad en función de objetivos generados a partir de necesidades sentidas y de acuerdo con estrategias colectivamente definidas, fundamentadas en la solidaridad y en el apoyo social" (Montero, 2003: 66). Una acción conjunta y solidaria que permita la inclusión democrática, la justicia distributiva y la autodeterminación, en donde no sólo se genera un involucramiento de los agentes sociales en problemáticas de su propio entorno sin esperar a que otro las resuelva por él, sino un fortalecimiento de la estructura social, enmarcando a las organizaciones en las que participa y ejecutando una política social de transformación real. Para dar cuenta de este proceso, es necesario exponer el contexto en el que se encuadran. 
Es en escenarios desfavorables para los sectores sociales más vulnerables, que la comunicación y las organizaciones comunitarias toman un rol clave en la dinámica social para cubrir demandas insatisfechas. Por ejemplo, en la Argentina, en contextos de neoliberalismo o de crisis institucional, como en 2001, donde las políticas públicas perjudicaron en todo sentido al sector popular, se genera además de una crisis económica y de representatividad política, una fragmentación social que lleva a los barrios e instituciones de los sectores populares a generar recursos para subsanar dicha crisis. La comunicación comunitaria pasa a tomar el rol de abarcar un escenario de disputa política entre los diversos actores de la comunidad, en donde el objetivo es sumar la mayor cantidad de agentes en la discusión, el debate y la solución participativa. Podemos decir, entonces, que:

\footnotetext{
La comunicación comunitaria es el intento de darle sentido a los elementos de la comunicación barrial y familiar desde la perspectiva de la posibilidad de la unión, la solidaridad y la organización del barrio. Está hecha de esos modestos pero permanentes impulsos solidarios en torno a la salud, la nutrición, la vivienda, la educación, que buscan el encuentro, la "colaboración" de los vecinos, la fiesta y el trabajo (Balán et al., 2013: 21).
}

De esta manera, en la dinámica comunitaria en cuanto al servicio del fortalecimiento barrial, popular, local, nos parece clave rescatar una de las organizaciones sociales relevantes en el fomento de la participación y el desarrollo comunitario en América Latina: las radios populares, comunitarias y alternativas. ¿Por qué la radio? Las radios comunitarias en Argentina se forman en contextos sociopolíticos de ausencia estatal en la solución de demandas sociales de los más desfavorecidos. Como centros de organización barrial, las radios comunitarias exponen las demandas y canalizan los problemas de su entorno, acompañando una dinámica de participación y fortalecimiento de sectores que fueron históricamente relegados por el aparato estatal. Como afirma María Cristina Mata: "constituyeron un espacio clave para la indignación de carácter ideologista, cuya finalidad era desmontar los mecanismos de dominación, las múltiples maneras de encubrir y falsificar la realidad, de manipular la pobre conciencia de los receptores" (1988: 66). Es un medio de comunicación económicamente accesible, popular y que no necesita una convención comunicacional más que el idioma oral para funcionar. Mientras que los medios masivos de comunicación tienen como fin el consumo de información y la propaganda institucional promoviendo conductas comerciales, los medios de comunicación comunitarios, como la radio, tienen como motor a la misma comunidad a la que se dirigen, generándose un círculo dinámico de comunicación participativa en donde "los receptores no son el último tramo de un proceso iniciado por el emisor, sino parte activa de él, tanto como productores de sentido pero también porque están presentes, inscriptos en el discurso del emisor" (Balán et al., 2013: 28). Para dar cuenta de la necesidad de estos espacios para el 
desarrollo social, expondré la injerencia del periodista y del comunicador comunitario como facilitadores de las dinámicas anteriormente expuestas.

\title{
El comunicador comunitario y el periodista
}

En este apartado observaremos dos prácticas profesionales de la comunicación como la del periodista y la del comunicador comunitario, que no son antagónicas, pero sí poseen diferencias sustanciales. Carlos Marín define la figura del periodista en Manual de Periodismo (2003) desde las diferentes actividades a la hora de construir una noticia:

\footnotetext{
Al que hace y redacta notas y entrevistas informativas, el reportero; al que elabora artículos, articulista; al que hace editoriales, editorialista; al que hace columnas, columnista; al que elabora caricaturas, caricaturista; al que toma fotografías, filmaciones o videos, reportero gráfico y al que ejercita la nota, cronista (2003: 23).
}

Entre los matices que existen entre el periodista y el comunicador comunitario, el reportero es el único que, en la descripción de sus tareas, tiene un contacto más real con el contenido de la noticia, los testigos y lo que sucede. Se lo define siempre desde la arista del periodista y la capacidad que debe tener el mismo para sacar la información necesaria que le permita construir una noticia verosímil y objetiva. Marín afirma que para el completo desempeño del periodista, hay que dar cuenta de "lo que le interesa a la gente" (2003: 45), y que termina siendo una incógnita sin solución ya que "la gente" es un concepto tan amplio como efímero. Esto nos da una primera aproximación sobre a qué público apuntan. Con el armado de la noticia, el periodista tiene que dirigirse a grandes masas de personas sin importar tanto el quién sino el cuánto. Por ejemplo, Marín afirma que "es el periodismo que entiende "lo popular" como algo que atañe a las mayorías, aunque el mensaje propiamente no sea leído o escuchado por la mayoría de la población" (2003: 47), haciendo hincapié en la variable cuantitativa, sin hacer foco en el entramado del campo popular en sí (4).

El comunicador comunitario tiene:

\begin{abstract}
una mirada más integral de la comunicación, relacionada con la condición esencial del ser humano que vive en comunidad, que se constituye como actor de manera relacional, que genera redes y procesos de organización basados en intercambios conversacionales y que, mediante la producción colectiva de sentidos, va constituyendo y construyendo la cultura que la contiene y que, al mismo tiempo, lo forja de manera característica (Uranga, 2016: 17).
\end{abstract}


La intervención que realiza el comunicador comunitario es entendida por Uranga (2016) como una acción política, que tiene como fin la transformación de la comunidad en la que se interviene, de modo que la misma pueda fortalecer sus vínculos organizativos y sociales, y por sobre todo su identidad como comunidad, sin ponderación de actores. La intervención en clave de acción política la entendemos bajo el paraguas del uso de la comunicación como herramienta para esa transformación. El comunicador comunitario vive en la notica, vive con la noticia; intenta construirla de manera relacional, dialógica y no de manera unilateral o vertical.

Como mencionamos en el primer apartado, la comunicación comunitaria surge en contextos donde los sectores populares necesitan de factores externos al estatal para atender las demandas y problemáticas más urgentes en el plano local, que no son atendidas por el mismo Estado. Hay escenarios en donde la comunicación habilita procesos de constitución de actores en democracia: "sujetos políticos, parciales, limitados, reales, que asuman los costos y las contradicciones de mantener las oportunidades abiertas, y sostener proyectos de una sociedad más justa" (Balán et al., 2013: 11). El comunicador comunitario surge como figura en periodos democráticos sumando relevancia a la participación y discusión de problemáticas locales que no pueden esperar la solución de aparatos estatales desbordados o sin interés.

Si bien "lo comunitario" puede ser analizado desde diversas aristas, en nuestro caso lo retomamos desde la mirada territorial, en donde el territorio se convierte en el campo de acción en el cual suceden las significaciones posteriormente analizadas. Tomando al barrio como contexto frecuente de acción del comunicador comunitario, hay ciertos elementos claves que lo conforman si lo analizamos comunicacionalmente:

1. Los actores, en tanto productores de los hechos comunicacionales.

2. Los espacios y circuitos (visuales, audiovisuales, auditivos y radiales).

3. Lugares de circulación.

4. Lugares de concentración, como espacios donde se reúnen los vecinos a lograr ciertos objetivos (Balán et al., 2013: 22).

Estas categorías son esclarecedoras para entender la dinámica de un barrio y con ellas hay que trabajar de manera conjunta en pos de transformar y fortalecer la organización local. Aquí, la intervención de un comunicador comunitario es pertinente para el desarrollo sostenido de los elementos mencionados. Hipotéticamente podemos dar cuenta de un adecuado relevamiento y diagnóstico del lugar por parte del periodista, pero en estos casos observaríamos la necesidad de integrar una intervención práctica, participativa y prolongada en el tiempo que ayude a las comunidades locales, a los barrios, a funcionar entre y por ellos mismos; o por lo menos a poder subsanar ciertos problemas cuando los haya, sin esperar de brazos cruzados la ayuda 
del Estado. La variable temporal es clave para entender al proceso comunicacional como una construcción estable y anclado al territorio y no como un simple acto de comunicación aislado. A diferencia de lo que puede ser la dinámica de trabajo del periodista, en tanto ir al campo, recopilar información, escribir la noticia y publicarla en algún medio; el trabajo del comunicador comunitario con los receptores pasa a cubrir un rol más interactivo, dinámico y participativo, siendo un nexo clave entre los mismos y su contexto. Creemos sumamente relevante poder contar con una reciprocidad continua, donde la información que fluye sea multidireccional "para que sea posible la retroalimentación informativa" (Sánchez, 2002: 110). Como afirmamos anteriormente, es necesario contar con un receptor activo en la producción de sentido.

Aquí es cuando nos parece pertinente el funcionamiento de medios comunitarios, como por ejemplo las radios, como nodos generadores de participación comunitaria y organización de los sectores locales frente a los problemas más urgentes. A partir de los estudios de casos expuestos en el tercer apartado, podremos dar cuenta del "trabajo en red, la flexibilidad necesaria para poner el medio al servicio de una causa, la labor sistemática en torno a problemas claramente definidos" (Gutiérrez y Mata, 2001: 76). Es a través de este tipo de medios que los comunicadores comunitarios trabajan en conjunto con la comunidad en pos de un fortalecimiento y progreso de la misma.

Es dentro de la especificidad de la tarea del comunicador comunitario, con su mirada más integral de la comunicación e intervención social, que se facilita un espacio de diálogo y construcción identitaria. Como menciona Uranga (2016) es en el modo de entender y de entenderse, de las formas de disputa y la manera en que se otorga sentido a aquello que llamamos realidad (muchas veces sesgada por coyunturas políticas), que se produce el conocimiento necesario para la intervención como acción política y la transformación de la comunidad.

Podemos afirmar que la participación es clave para el funcionamiento de lo antes expuesto. Pero es evidente, también, que es necesario el acompañamiento de liderazgos sociales que aseguren conjuntamente todo el proceso. Además del objetivo de solución de problemas inmediatos, la intervención comunitaria en un contexto participativo lleva como gran objetivo la construcción de fortalecimiento definida por Montero como:

\footnotetext{
El proceso mediante el cual los miembros de una comunidad (individuos interesados y grupos organizados) desarrollan conjuntamente capacidades y recursos para controlar su situación de vida, actuando de manera comprometida, consciente y crítica, para lograr la transformación de su entorno según sus necesidades y aspiraciones, transformándose al mismo tiempo en sí mismos (2003: 72).
}

Si bien son dinámicas y procesos ideales de transformación e intervención comunitaria, podemos dar cuenta que para que esto se genere, es de indispensable necesidad la 
participación conjunta de la sociedad. Entre mayor participación e involucramiento de la comunidad, más se garantiza la inclusión de todas las visiones y problemáticas; generando lazos más fuertes y una identidad de base para impulsar innumerable cantidad de proyectos.

\section{Estudio de caso: radio Encuentro (Viedma)}

Acontecimientos como el Concilio Vaticano II (1959) (5), las Conferencias Generales del Episcopado Latinoamericano, desarrolladas en Medellín (1968) (6) y luego en Puebla (1979) (7), desarrollan tendencias renovadoras y progresistas en la cúpula eclesiástica católica, que derivan en la formación de sacerdotes con gran vocación de servicio y participación con su comunidad. A partir de estos acontecimientos puede comprenderse el trabajo comunitario realizado por el obispo Jaime de Nevares en Neuquén y el obispo Miguel Esteban Hesayne en Viedma, Río Negro.

En el caso de Jaime de Nevares, es reconocido su trabajo con los sectores populares en formación, desde la década del 80. Entre estos trabajos, podemos destacar la formación del Servicio para la Comunicación y Capacitación (SERPAC), en donde se gesta la radio comunidad Enrique Angelelli, actualmente en funcionamiento. Desde la participación comunitaria, hasta la formación en comunicación, la radio comunitaria iniciada por el obispo pasó a ser un centro de formación de comunicadores comunitarios que incidieron invaluablemente en el fortalecimiento de la comunidad neuquina y la comunicación popular patagónica (8).

Bajo el mismo proceso formativo antes descripto e impulsado por la experiencia comunicacional neuquina, el obispo de la ciudad de Viedma Miguel Hesayne, junto a su compañero Néstor Busso, decidieron inaugurar la radio Encuentro en 1989. Al igual que el proyecto iniciado por Jaime de Nevares en Neuquén, Encuentro nació luego de una "revista que salía semanalmente para poder visibilizar los sectores populares y a ese proyecto se le sumó el de la posibilidad de hacer una radio" (Cabral, 2017). Ya con la radio funcionando dentro del Obispado, se crea la Asociación Alternativa en Comunicación Social, que hasta hoy es propietaria de la radio.

Néstor Busso estuvo exiliado en Brasil a raíz de la última dictadura militar y allí pudo instruirse, entre otras cosas, en la pedagogía de la liberación de Freire (9). Una vez vuelto a Viedma, se sumó al proyecto impulsado por el obispo Hesayne y nunca abandonó su rol protagónico, hasta el día de hoy que sigue siendo el presidente de la asociación, como lo fue del Foro Argentino de Radios Comunitarias (FARCO), entre otros organismos. Desde la misma asociación que nucleaba radio Encuentro (10), Busso y sus colaboradores llevaron la lucha por un espacio en el marco legal que llegó a la corte suprema de justicia: 
Nosotros fuimos, junto con la radio "La Ranchada" de Córdoba, que hicimos juicio hasta la Corte Suprema con el decreto ley de la década del 90. El decreto que regía las comunicaciones antes de la ley sancionada en 2009, que prohibía a las organizaciones sociales ser propietarias de medios, que era el artículo 45 (M. C. Cabral, comunicación personal, 16 de enero de 2017).

Existen tres aspectos fundamentales que queremos destacar de radio Encuentro con respecto a lo que venimos analizando en relación a la importancia de la comunicación comunitaria para el desarrollo y fortalecimiento de las comunidades. La primera es la destacable adecuación práctica y resolutiva que tuvo la radio según los diversos contextos. La segunda viene de la mano de veta periodística y comunicacional característica de Encuentro y la tercera, y a modo de conclusión del apartado, la vocación de liderazgo que construyeron desde la radio Encuentro a lo largo de los años.

En los primeros dos apartados rescatábamos la importancia de los medios de comunicación comunitaria en contextos sociopolíticos adversos, donde la figura estatal queda desdibujada. En el caso de Encuentro, María Cristina Cabral nos comenta las diversas etapas que tuvo la asociación, entre las que resalta la década neoliberal de los años 90, cuando los sectores populares eran dejados de lado ante las políticas privadas y comerciales:

En la década de los $90^{\prime}$ fue una radio muy centrada en lo que fueron las protestas sociales. Acá en Río Negro, en el 95' estalló la provincia. Era gobernador Massacchesi, que después fue candidato a presidente de la Nación, así que fue una radio que siempre tuvo incidencia; primero en los barrios: todas las mañanas se traían las noticias de lo que pasaba en los barrios y se llevaban las consultas a los funcionarios (M. C. Cabral, comunicación personal, 16 de enero de 2017).

Cabe destacar que, para esta época, es posible identificar un auge de radios comunitarias en el país a partir de los diversos atropellos estatales que sufrió el sector popular. Por otra parte, legalmente se seguía funcionando bajo la ley de la última dictadura militar que ni siquiera contemplaba a los medios de comunicación provenientes de organizaciones sin fines de lucro, otra lucha que llevó a cabo radio Encuentro, relatada anteriormente.

Cabral nos cuenta que en los años 2000 también se acompañó mucho a la protesta social, inclusive hasta la primera etapa del kirchnerismo, cuando avanzada la gestión se pudo ver un acompañamiento estatal a partir de reivindicaciones históricas como en la temática de derechos humanos y la legislación laboral, entre otras cosas. Desde ese momento se pudieron ver en los barrios de Viedma organizaciones sociales que surgían y eran articuladas bajo el ala 
de radio Encuentro. Podemos destacar, a partir de los relatos mencionados, el papel preponderante de la radio Encuentro en el acompañamiento de los sectores sociales ante reclamos que no eran atendidos por el Estado.

Cabral nos destaca que radio Encuentro:

Tiene un perfil periodístico (11) desde sus inicios, es algo que la distingue de otras radios comunitarias en Argentina y también en la formación, porque se creía que se tenía que formar a los sectores populares y a las organizaciones populares para que puedan comunicar ellos mismos sus necesidades y organizar el campo popular (M. C. Cabral, comunicación personal, 16 de enero de 2017).

Es aquí donde podemos observar un cruce de todo lo planteado hasta ahora. La notable incidencia que tuvo y tiene la radio en relación al diálogo con su comunidad, la participación otorgada y el fortalecimiento generado, reflejado en la incipiente formación de organizaciones sociales, todo lo cual ayudó a que se conectasen entre sí. Pero no es sólo guiar a la organización de la comunidad desde la comunicación comunitaria, sino que desde la radio incluyen la formación periodística para que la misma comunidad pueda dar cuenta de lo que sucede en su propio entorno y plasmarlo en diversos soportes, sin tener que esperar a que un medio de comunicación externo imponga su mirada, pudiendo dar cuenta de una coparticipación del periodismo y la comunicación comunitaria con desarrollos más que fructíferos.

Es así como desde el centro de formación de Viedma, ubicado en las instalaciones de la radio Encuentro, con ayuda del Foro Argentino de Radios Comunitarias (FARCO) y el plan PROGRESAR (12), se dictan cursos de "nuevas tecnologías en radio", enmarcados en la sanción de la Ley de servicios de comunicación audiovisual, sancionada en 2009, para ayudar a comprender el nuevo campo de las comunicaciones ante la posibilidad de democratización del espectro radiofónico que propone la ley. Otro curso dictado es el de "producción periodística", que propone formar a comunicadores y periodistas en tanto críticos de la realidad, pudiendo dar cuenta de la agenda informativa que sucede a su alrededor y adquirir las herramientas necesarias para visibilizar la misma. Un tercer curso dictado es el de "artística y creatividad en radio", centrado en el perfeccionamiento del trabajo radial, con el fin de poder darle una impronta personal al trabajo que se realiza.

Es necesario destacar la tarea que lleva realizando radio Encuentro, ya no sólo en el acompañamiento de las demandas sociales, sino también desde la difusión constante de información que no era incluida por los medios de comunicación privados y desde la instrucción y capacitación de miembros de la comunidad, para que posean un criterio propio ante los acontecimientos cotidianos en su entorno. 
Un tercer aspecto referido a la radio Encuentro es la destacable vocación de liderazgo con respecto a otras organizaciones sociales de la región, por un lado, y en relación a las demás radios comunitarias del país, por otro. Mata afirma de la radio Encuentro que:

Podemos compartir o no la forma cómo se trabaja a partir de esa vocación, pero allí hay vocación de liderazgo. Y para mí lo que está claro es que uno no puede dar batallas en el espacio público para generar ciertas ideas y opiniones si no se desea liderar un movimiento o corriente de opinión (Gutiérrez y Mata, 2001: 39).

Habiendo expuesto sus inicios, su dinámica de diálogo y participación con su comunidad y la iniciativa de formación comunitaria en periodismo y comunicación, deja en evidencia una posición de liderazgo e iniciativa de Encuentro que da pie no sólo al fortalecimiento y desarrollo de la comunidad, sino también a la generación de nuevos medios de comunicación comunitarios en la región y el país, ayudando al fomento de un modo alternativo de llevar a cabo la comunicación cotidiana y duradera (13).

\section{Reflexiones finales}

A lo largo del trabajo fuimos desentrañando desde lo macro de la comunicación, en tanto factor trasversal de nuestra vida cotidiana, hasta la comunicación comunitaria como un modo de trabajar desde una veta más participativa, local y necesaria en el desarrollo de organizaciones y comunidades populares, muchas veces relegadas en el plano macro; para finalizar en un caso ejemplificador como el de radio Encuentro, que nos permitió dar cuenta de todo lo antes expuesto.

Pasamos luego a los actores que trabajan activamente en el desarrollo comunicacional de la sociedad, proponiendo una comparación entre las categorías de periodista y comunicador comunitario, haciendo foco en su rol profesional. Aquí hemos seguido la lógica del primer apartado, yendo de lo macro a lo micro en relación al trabajo del periodista y el trabajo profesional de armado de la noticia, desde el momento de investigación hasta que sale en algún canal de comunicación; y el trabajo sostenido del comunicador comunitario en espacios sociales más acotados, que apunta a la mejora en la calidad organizativa de una comunidad, reflejado luego en el fortalecimiento y empoderamiento de la misma. El cruce teórico propuesto también nos invita a rescatar que la práctica periodística no tiene fin en sí misma, sino un entramado de sentidos que debemos dar cuenta.

Para poder anclar los dos primeros apartados hemos utilizado el caso de radio Encuentro, que nos posibilitó darnos cuenta de la importancia de la comunicación en la vida cotidiana de los habitantes de Viedma y cómo el trabajo sostenido y perseverante la llevó a ser un medio 
reconocido, no sólo por la comunidad sino por otras organizaciones de la misma índole que pudieron seguir su ejemplo comunicacional y social.

Queda claro que las categorías de comunicación, comunicación comunitaria, así como la de periodista y comunicador comunitario no son puras y cerradas. Con el ejemplo de Encuentro pudimos ver cómo ambas pueden convivir sin ser contrapuestas; se complementan para promover el diálogo de la comunidad e incentivar a que los miembros de la misma puedan participar en la construcción de su propio entorno, en pos de la transformación social. Claramente la apropiación de la información que nos rodea, canalizada por una vía de fortalecimiento y desarrollo, son dinámicas que deben ser trabajadas por agentes que puedan acompañar este proceso.

Finalmente, nos parece insuficiente llegar al punto que esos agentes tengan que hacer el "trabajo" y servir esa información para ser consumida sino, al contrario, poder dar las herramientas necesarias para que los mismos "receptores" o integrantes de esa comunidad puedan apropiarse y resignificar en acciones concretas la comunicación, que de por sí ya nos atraviesa.

\section{Notas}

(1) Ley de Radiodifusión 22285, sancionada en marzo de 1980 bajo el gobierno de facto de Jorge Rafael Videla.

(2) Los medios comunitarios pasan a ser incluidos como emisores y participantes del debate de la ley. Se les asignan frecuencias $y$ fondos a algunas emisoras.

(3) Utilizamos el concepto de "manipulación" desde lo trabajado por Armand Mattelart (1997) en relación a la explicación de empirismos del nuevo mundo, procesos homogeneizadores de los medios de comunicación así como individualidades que permiten abstraerse y construir su entorno.

(4) Si bien entendemos que existen numerosas definiciones de "periodista", la expuesta nos permite retomar la variable del destinatario, clave en la dinámica de comunicación en tanto mediaciones más que de medios.

(5) Concilio convocado por el Papa Juan XXIII en 1959 y finalizado en 1965 por el Papa Pablo VI, discutiendo aspectos estructurales de la iglesia católica.

(6) Segunda conferencia episcopal, precedida por el Papa Pablo VI y se debatió como poner en práctica lo trabajado en el Concilio Vaticano II.

(7) Tercer conferencia episcopal que se centra en el debate de la pobreza, los jóvenes y la construcción de la pluralidad en América Latina y el Caribe.

(8) Para un desarrollo más amplio del proyecto comunicacional popular promulgado por Jaime de Nevares en la capital neuquina.

(9) También enriquecida con los acontecimientos episcopales mencionados en un principio del apartado.

(10) Fundación en Comunicación Alternativa y Popular.

(11) Nos referimos a perfil periodístico por agenda propia de noticias y generación de información. Dar la palabra a ONG's, organizaciones barriales, de derechos humanos, colectivos de mujeres, etcétera. Referentes de la información tanto desde la generación como actores. Un periodismo desde y para la comunidad.

(12) http://www.progresar.anses.gob.ar/ 
(13) Por ejemplo también con el vínculo, desde 2010, con la Universidad Nacional de Río Negro en el dictado de cursos radiales para licenciado y docentes interesados.

\section{Bibliografía}

Balán, E.; Jaimes, D.; Alegría, H. y Borri, N. (2013). Barrio Galaxia. Manual de la comunicación comunitaria. Ciudad de Buenos Aires, Argentina: Centro Nueva Tierra.

Cardoso, N. (2006). Pensar los medios de comunicación para las organizaciones desde una perspectiva participativa y comunitaria. Ficha de cátedra Comunicación Comunitaria. Carrera de Ciencias de la Comunicación. Universidad de Buenos Aires.

Gutiérrez, H. y Mata, M. C. (2001). ¿Siguen vigentes las radios comunitarias? Quito, Ecuador: Asociación Latinoamericana de Educación Radiofónica.

Habermas, J. (1981). Teoría de la acción comunicativa I. Racionalidad de la acción y racionalización social. Alemania. Ed. Taurus.

Marín, C. (2003). Manual del Periodismo. México: Ed. Grijalbo.

Martín-Barbero, J. (1987). De los medios a las mediaciones. Barcelona: Ediciones Gili.

Mata, M. C. (1988). Radios y públicos populares. Revista Diálogos, 19, p. 59, La Plata, Argentina.

Mata, M. C. (1993). La Radio: una relación comunicativa. Revista Diálogos, 35, La Plata, Argentina.

Mattelart, A. y Mattelart, M. (1997). Historia de las teorías de la comunicación. Buenos Aires. Argentina: Ed. Paidós.

Mac Bride, S. (1980). Un solo mundo, voces múltiples. Comunicación e información en nuestro tiempo. México: Fondo de cultura económica.

Montero, M. (2003). El fortalecimiento en la Comunidad en Teoría y práctica de la psicología comunitaria. Buenos Aires, Argentina: Editorial Paidós.

Postolsky, G. y Marino, S. (2005). Relaciones peligrosas: Los medios y la dictadura, entre el control, la censura y los negocios. En Mucho ruido, pocas leyes (pp. 156-188). Buenos Aires. Argentina: Ed. La Crujía.

Sánchez, M. (2002). La psicología social de la comunicación. Sus aplicaciones en el aula. En Sánchez Marín, M.; Grau Gumbau, R. y Yubero Jiménez, S. Procesos Psicosociales en los contextos educativos. Madrid, España: Ediciones Pirámide.

Saintout, F. y Varela, A. (2014). La epistemología del barro. Revista Oficios terrestres, 32, pp 109-117.

Uranga, W. (2016). Conocer, transformar, comunicar. Ciudad de Buenos Aires, Argentina: Editora Patria Grande. 
Uranga, W. y Thompson, H. (2016). La Incidencia como camino para la construcción de ciudadanía. Una propuesta para trabajar desde la comunicación. Ciudad de Buenos Aires, Argentina: Editora Patria Grande. 